\title{
AVALIAÇÃO DA ATENÇÃO BÁSICA: A OUVIDORIA ATIVA COMO ESTRATÉGIA DE FORTALECIMENTO DO CUIDADO E DA PARTICIPAÇÃO SOCIAL
}

\section{EVALUATION OF PRIMARY CARE: A CALL ACTIVE AS STRENGTHENING CARE STRATEGY AND THE SOCIAL PARTICIPATION}

\section{Hêider Aurélio Pinto}

Mestre em Saúde Coletiva pelo

Programa de Pós-Graduação em

Saúde Coletiva da UFRGS. Secretário

de Gestão do Trabalho e da

Educação na Saúde do Ministério da Saúde.

E-mail: heider.aurelio.pinto@gmail. com

\section{Andréia Burille}

Professora da Universidade do vale do Rio dos Sinos. Doutorando em Enfermagem na UFRGS.

E-mail: andreiaburille@yahoo.com. br

\section{Mirceli Goulart Barbosa}

Mestre em Saúde Coletiva pelo Programa de Pós-Graduação em Saúde Coletiva da UFRGS; Especialista em Residência Integrada em Saúde pelo Grupo Hospitalar Conceição; Educadora física e nutricionista, coordenadora técnica do PMAQ/Rede Governo Colaborativo em Saúde (UFRGS). E-mail: mirceligoulart@yahoo.com. br

\author{
Alcindo Antônio Ferla \\ Médico, Doutor em Saúde Coletiva, Professor do \\ Programa de Pós-Graduação em Saúde Coletiva \\ da UFRGS, Coordenador Nacional da Rede Unida \\ e Coordenador da Rede Governo Colaborativo em \\ Saúde (UFRGS). \\ E-mail: ferlaalcindo@gmail.com
}

\section{Resumo}

Objetivo: Refletir sobre os dados divulgados pelo "Relatório de Pesquisa de Satisfação com cidadãos usuários e não usuários do Sistema Único de Saúde (SUS)", elencados pelo dispositivo de Ouvidoria Ativa em Saúde. Métodos: Apresentase uma análise crítica-reflexiva a partir de dados secundários, apresentados em 3 eixos: 1) quem são os usuários do SUS; 2) Atenção Básica (AB)referência do cuidado; 3) Satisfação com o SUS. Resultados: Dentre os 26.692 usuários ouvidos pela pesquisa, 70\% mencionaram a utilização do SUS, em contrapartida a $30 \%$ que não utilizaram estes serviços. Em relação ao perfil dos entrevistados, evidencia-se no grupo que utilizou nos últimos 12 meses o serviço de $A B$ ou de urgência do SUS que $77 \%$ das pessoas entrevistadas não possuem plano de saúde. Dentre os entrevistados que responderam ter utilizado algum serviço do SUS, 
85\% deles haviam sido atendidos nas Unidades Básicas de Saúde (UBS). Dentre aqueles que buscaram algum serviço do SUS, 36\% atribuíram o conceito de muito bom ou bom, $34 \%$ de regular, seguido de $29 \%$ que consideraram como ruim ou muito ruim. No grupo composto por entrevistados que não acessaram nenhum serviço do SUS nos últimos 12 meses os percentuais ficaram em $25 \%$ com avaliação muito boa ou boa, $30 \%$ como regular e $45 \%$ como ruim ou muito ruim. Conclusões: O panorama elucidado mostra avanços significativos em relação ao SUS, e em especial, ao que corresponde à $A B$. Cabe destacar a potência da Ouvidoria Ativa no fortalecimento do SUS e na operacionalização dos seus princípios, diretrizes e serviços.

Palavras-chave: ouvidoria dos pacientes; atenção básica à saúde; Sistema Único de Saúde.

\section{Abstract}

Objectives: Reflect on the data released by the "Satisfaction Survey Report with citizens users and non users of the Unified Health System (UHS)", listed by the Ombudsman Active device in Health. Methods: It presents a critical - reflexive analysis based on secondary data, presented in three areas: 1 ) who are the users of UHS ; 2 ) Primary Care (PC) - Reference care ; 3) Satisfaction with the UHS. Results: Among the 26692 users polled by the survey, $70 \%$ mentioned the use of UHS, in contrast to $30 \%$ who did not use these services. Regarding the profile of respondents, is evident in the group that used the past 12 months the PC service or UHS urgency that $77 \%$ of respondents have no health insurance. Among the respondents who reported having used some of the UHS service, $85 \%$ of them had been met in the Basic Health Units (BHU). Among those who sought some UHS service, $36 \%$ attributed the concept of good or very good, $34 \%$ fair, followed by $29 \%$ who considered it as bad or very bad. In the group of respondents who have not accessed any service UHS in the last 12 months, the percentages were $25 \%$ with very good or good rating , $30 \%$ as fair and $45 \%$ as poor or very poor. Conclusions: The outlook elucidated shows significant progress in relation to UHS, and in particular, corresponding to the PC. It should be noted the power of Active Ombudsman to strengthen the UHS and in the operationalization of the principles, guidelines and services.

Keyword: patient advocacy; primary health care; Unified Health System.

\section{Introdução}

A reorganização da atenção em saúde, alicerçada nos princípios da universalidade, integralidade, equidade, intersetorialidade, humanização e controle social, constitui um desafio cotidiano para todos os atores que militam no Campo da Saúde Coletiva. ${ }^{1}$ Justificam-se esses esforços, na medida em que, a complexidade de apreender a diversidade de condições e situações que permeiam o adoecer e o ser saudável, tornam a tarefa de ofertar modelos de cuidados efetivos, uma ação constante e necessária para a consolidação do acesso e da qualidade a saúde para todos os brasileiros.

$\mathrm{Na}$ trajetória do Sistema Único de Saúde (SUS), diversas propostas têm sido implementadas, em especial, no âmbito municipal, no qual a partir da metade da década de 90 , vem se mobilizando esforços 
em prol da reorganização da Atenção Básica (AB). Por contemplar um conjunto de ações que vão desde a promoção de práticas saudáveis até tratamentos e medidas de reabilitação, esse modelo de atenção apresenta potencial significativo no cuidar de indivíduos, famílias e comunidades, sendo alvo de múltiplas estratégias, entre elas, a Portaria 2.488 de 21 de outubro de 2011, que define a $A B$ como porta de entrada e ordenadora do acesso universal e igualitário aos serviços da Rede de Atenção à Saúde (RAS). ${ }^{2}$

Oreconhecimento das potencialidades e a oferta de mecanismos que possibilitem melhor gestão do cuidado no âmbito da $A B$ apresentam-se como estratégias factíveis e robustas, que ganham contornos especiais quando contemplam práticas avaliativas na perspectiva de usuários. 0 acompanhamento dessas experiências vem se dando por meio de taxas de cobertura e indicadores epidemiológicos, que se mostram insuficientes para perceber os movimentos de mudança na qualidade de atenção, principalmente no que se refere ao processo de trabalho das equipes, na relação com a população adstrita e a integralidade da atenção à saúde. ${ }^{1}$

Corriqueiramente, observa-se nas práticas avaliativas a não centralidade no usuário a partir do que ele pensa e vive, mas a partir de como esse serviço o vê. Está presente a lógica do serviço, que leva em conta indicadores de desempenho, sem considerar as dimensões subjetivas, o que por sua vez dificilmente irão dar conta da complexidade da vida cotidiana e das suas repercussões na saúde. ${ }^{3}$

Os estudos relacionados à satisfação dos usuários iniciaram na década de 1970, influenciados pelo paradigma do consumismo e pela cultura da qualidade em países como Inglaterra e Estados Unidos. ${ }^{4}$ No Brasil, essa inspiração surgiu na década de 90, devido ao fortalecimento da participação social no âmbito do SUS. Nesse contexto, as avaliações na perspectiva do usuário, estavam vinculadas aos conselhos de saúde, que em diferentes esferas de gestão, representavam um loccus, nos quais diferentes atores poderiam expressar suas inquietações e posicionamentos.

Esses espaços, nos quais os usuários assumem protagonismo no "fazer saúde" têm se mostrado importantes vetores para melhoria do acesso e da qualidade de atenção. Nesse sentido, várias apostas vêm sendo realizadas nas Ouvidorias em Saúde, que representam dispositivos capazes de viabilizar a participação dos usuários. Todavia, há que se destacar que não basta cria apenas canais de acesso para manifestações, é preciso uma ouvidoria ativa, pela qual se produz estratégias de escuta do usuário através da busca ativa de informações. ${ }^{5}$

O movimento de ouvidoria ativa, além de viabilizar a consolidação do princípio da participação social, consegue apreender como os usuários experienciam, representam ou constroem imaginários sobre os serviços de saúde no nível micro e no macro, sobre o SUS. Assim, considerando o cenário aqui elucidado, esse manuscrito propõe-se a refletir sobre os dados divulgados pelo "Relatório de Pesquisa de Satisfação com Cidadãos Usuários e não Usuários do SUS", elencados pelo dispositivo de Ouvidoria Ativa em Saúde.

\section{Metodologia}

Apresenta-se uma análise críticareflexiva de dados referentes à $A B$ publicado ${ }^{i}$ no "Relatório de Pesquisa de Satisfação com Cidadãos Usuários e não Usuários do SUS quanto aos aspectos de acesso e qualidade percebida na atenção a saúde ${ }^{\mathrm{i} i}$, , realizada

\footnotetext{
Dados Secundários.

ii Disponível em: <http://portal.saude.gov.br/portal/ arquivos/pdf/relatorio_da_pesquisa_de_satisfacao.
} 
pelo Departamento de Ouvidoria Geral do SUS $^{6}$, com 26.662 participantes, distribuídos em todo território nacional, nos períodos de junho a julho de 2011 e novembro de 2011 a janeiro de 2012 .

Os dados supracitados foram gerados por meio de contato telefônico assistido por computador, a partir de bases de dados fornecidas por empresas de telefonia. Após serem informados sobre o objetivo da pesquisa e o órgão executor, 18.673 usuários que mencionaram que utilizaram o SUS nos últimos 12 meses antes da entrevista, responderam um questionário de 41 perguntas divididas em quatro partes: atenção básica; atenção odontológica; urgência e emergência; perfil. Para quem não possuía histórico de utilização do SUS no período citado (7.989 usuários), além do perfil, os demais questionamentos visaram identificar a opinião quanto ao SUS e acesso a outras formas de assistência à saúde.

As análises expressas nesse manuscrito foram geradas após leitura do relatório de pesquisa. Com essa etapa concluída, os dados relacionados ao perfil dos entrevistados e satisfação com o SUS foram recortados, agrupados ${ }^{\text {iii }}$ e agregados em dois grupos: usuários e não usuários do SUS. Os dados referentes à avaliação da $A B$, gerados pelos entrevistados que referiram utilizar os serviços do SUS, também sofreram recortes e foram agrupados de acordo com os questionamentos. Após essa etapa elencou-se três eixos de apresentação: 1) quem são os usuários do SUS?; 2) Atenção Básica-referência de Cuidado; e 3) Satisfação com o SUS.

Com relação aos aspectos éticos, a discussão apresentada integra o projeto de pesquisa "Avaliação da atenção básica no Brasil: estudos multicêntricos integrados sobre acesso, qualidade e satisfação dos usuários", no que se refere ao objetivo de pdf>. Acesso em: 15 jan 2016.

iii No relatório os dados encontram-se separados por regiões. discutir e avaliar o acesso e a qualidade da qualidade da $A B$ em diferentes contextos, sendo aprovado no Comitê de Ética em Pesquisa da Universidade Federal do Rio Grande do Sul, sob protocolo no 21904.

\section{Resultados}

\section{Eixo 1- Quem são os usuários do SUS?}

Num primeiro momento, antes de problematizar e traçar o perfil dos usuários do SUS é importante destacar que entre os 26.692 ouvidos pela pesquisa, $70 \%$ (18.673) mencionaram a utilização do SUS, em contrapartida a 30\% (7.989) que não utilizaram os serviços em busca de consultas, exames, vacinação, atendimento de urgência, internação ou medicamentos, no recorte temporal de 12 meses.

Seguido desse primeiro recorte, podem-se constatar algumas diferenças entre os dois grupos (Tabela 1 ). Em relação ao perfil dos entrevistados, evidencia-se no grupo que referiu ter utilizado nos últimos 12 meses o serviço de $A B$ ou de urgência do SUS que $77 \%$ das pessoas entrevistadas não possuem plano de saúde. Observa-se também neste grupo, um maior percentual de mulheres e pessoas de cor parda. Além da maior utilização dos serviços por parte das mulheres, é possível observar fatores que marcam diferenças socioeconômicas importantes nesses dois grupos. Já no grupo que referiu não utilizar o SUS, evidenciase os dados referentes à renda familiar, pois neste grupo $47 \%$ recebe mais de dois salários mínimos, a escolaridade, tendo em vista que $19 \%$ possuem nível superior de escolaridade completo. 
Tabela 1- Diferenças entre os grupos entrevistados

\begin{tabular}{lcc}
\hline & $\begin{array}{c}\text { Utilizaram o } \\
\text { SUS }\end{array}$ & $\begin{array}{c}\text { Não utilizaram o } \\
\text { SUS }\end{array}$ \\
\hline Não têm Plano de Saúde & $77 \%$ & $49 \%$ \\
Sexo Feminino & $67 \%$ & $55 \%$ \\
Declaram-se Brancos & $41 \%$ & $45 \%$ \\
Declaram-se Negros & $12 \%$ & $11 \%$ \\
Declaram-se Pardos & $43 \%$ & $40 \%$ \\
Têm Nível Médio de Escolaridade Completo & $43 \%$ & $44 \%$ \\
Têm Nível Superior de Escolaridade Completo & $10 \%$ & $19 \%$ \\
Renda familiar maior que 2 salários mínimos entre os & $30 \%$ & $47 \%$ \\
que declararam & & \\
Renda familiar maior que 10 salários mínimos entre os & $1,3 \%$ & $4,7 \%$ \\
que declararam & & \\
\hline
\end{tabular}

Fonte: Relatório de Pesquisa de Satisfação com Cidadãos Usuários e não Usuários do SUS quanto aos aspectos de acesso e qualidade percebida na atenção a saúde-Departamento de Ouvidoria Geral do SUS (DOGES, 2012).

\section{Eixo 2- A Atenção Básica como referência de cuidado}

Não passa de uma década, o discurso e, consequentemente as ações em saúde, apontam para o entendimento de uma $A B$ acessível, responsável pelo acolhimento e primeiro contato dos usuários, com amplo leque de ofertas de ações de saúde, resolutiva e com capacidade de agregar as tecnologias necessárias para atender à maior parte possível das necessidades de saúde dos usuários perto de onde eles vivem. ${ }^{7}$ Portanto, entender como a população compreende o SUS é importante. A proposta de trabalhar a Ouvidoria em Saúde, como espaço potencial de avaliação dos serviços na perspectiva do usuário é fundamental para atingir este objetivo.

Dentre os entrevistados que responderam ter utilizado algum serviço do SUS (70\%), 85\% deles haviam sido atendidos nas Unidades Básicas de Saúde (UBS). Para esses usuários questionou-se também o nível de satisfação com os profissionais de enfermagem, médicos, ACS e cirurgiões dentistas.

Com relação à satisfação, entendida como expressão do "contentamento de um indivíduo com uma situação, serviço ou até outros indivíduos" ${ }^{\prime 6}$ pode-se evidenciar que $63 \%$ dos entrevistados avaliaram o atendimento de enfermagem como muito bom ou bom, e $72 \%$ verbalizaram esta percepção para os profissionais médicos. Sobre os Agentes Comunitários de Saúde, o questionamento foi realizado para $42 \%$ dos usuários, que disseram ter recebido a visita destes profissionais, sendo o percentual de satisfação em torno de $73 \%$. A avaliação dos cirurgiões dentistas foi realizada por $18 \%$ dos usuários que disseram ter buscado atenção odontológica nos últimos 12 meses, e as respostas satisfatórias foram mencionadas por $80 \%$ dos entrevistados. Assim, considerando que as avaliações positivas variaram de $63 \%$ a $80 \%$ para os quatro profissionais avaliados e os números de ruim e muito ruim não passaram de $10 \%$, pode-se afirmar, com estes e com os achados que seguem que o grau de satisfação com os profissionais de $A B$ é muito bom e inferir que as Unidades de Básicas de Saúde têm 
conseguido acolher os usuários e na medida do possível lhes ofertar certa resolubilidade. A tabela 2 apresenta os dados referentes à satisfação dos usuários, quanto à satisfação com o cuidado ofertado por estes profissionais.

Tabela 2- Satisfação dos usuários com os profissionais

\begin{tabular}{lccc}
\hline \multicolumn{1}{c}{ Profissionais } & Muito Bom + Bom & Regular & Ruim + Muito Ruim \\
\hline Enfermeiros & $63 \%$ & $26 \%$ & $10 \%$ \\
Médicos & $72 \%$ & $17 \%$ & $10 \%$ \\
Agentes Com. Saúde & $73 \%$ & $20 \%$ & $6 \%$ \\
Cirurgiões Dentistas & $80 \%$ & $12 \%$ & $8 \%$ \\
\hline
\end{tabular}

Nota: Os percentuais acima não totalizam $100 \%$ devido ao fato de uma parcela dos usuários não ter respondido à pergunta.

Fonte: Relatório de Pesquisa de Satisfação com Cidadãos Usuários e não Usuários do SUS quanto aos aspectos de acesso e qualidade percebida na atenção a saúde- Departamento de Ouvidoria Geral do SUS (DOGES, 2012).

O vínculo com Equipe Saúde da Família (ESF) foi mencionado por $55 \%$ dos usuários que referiram ter buscado cuidado em Unidades Básicas de Saúde, sendo que $42 \%$ destes receberam algum profissional de saúde em domicilio, pelo menos uma vez nos últimos seis meses. Importante destacar que os percentuais mencionados convergem para a estimativa de cobertura populacional pela ESF (54\%), gerados pelo Departamento de Atenção Básica do MS, no período pesquisado.

Com relação à distribuição das unidades básicas no território, o estudo apontou que $87 \%$ dos entrevistados responderam que demoram menos de 30 minutos para chegar a UBS mais próxima de casa. Esse dado, do ponto de vista da acessibilidade e das dimensões do território de referência, está de acordo com a nova Política Nacional da Atenção Básica (PNAB), publicada em 2011, que recomenda 3 mil pessoas para cada ESF e o máximo de 12 mil pessoas por UBS orientada por esta estratégia. Para UBS que não são orientadas pela ESF e que estão em áreas de grande concentração populacional recomenda-se o teto de até 18 mil pessoas. ${ }^{2}$

Perguntados se para serem atendidos precisaram agendar suas consultas previamente, $51 \%$ dos usuários responderam que conseguem agendamento no mesmo dia, 34\% referiu um período de uma semana a um mês e $13 \%$ mencionou mais de um mês. Já com relação ao tempo de espera na UBS, os dados apontam que $54 \%$ dos usuários aguardam menos de uma hora, em contrapartida, a $23 \%$ que mencionaram obter atendimento entre uma e quatro horas e 13\% que referiram ter aguardado mais de quatro horas ou não ter conseguido atendimento.

Ainda os entrevistados que utilizaram UBS responderam questões sobre demandas levadas, recomendação e avaliação do serviço (Tabela 3). Interessante notar que, embora a pesquisa tivesse como premissa associar satisfação com atendimento da demanda do usuário, os números revelam que ter a demanda atendida não significa necessariamente avaliar positivamente o serviço. O número de pessoas que tiveram a demanda atendida é de $60 \%$ e o número de pessoas que avaliam os serviços como bom e muito bom é de $38 \%$. 
Tabela 3- Recomendação, avaliação e atendimento das demandas na UBS

\begin{tabular}{ll}
\hline \multicolumn{1}{c}{ Entrevistados } & UBS \\
\hline Recomendaria & $74 \%$ \\
Não Recomendaria & $25 \%$ \\
Avalia o serviço como Muito bom/Bom & $38 \%$ \\
Avalia como Regular & $35 \%$ \\
Avalia como Ruim/Muito Ruim & $28 \%$ \\
Teve demanda atendida & $60 \%$ \\
Parcialmente atendida & $25 \%$ \\
Não teve demanda atendida & $14 \%$ \\
\hline
\end{tabular}

Nota: Os percentuais acima que não totalizam $100 \%$ devem-se ao fato de uma parcela dos usuários não ter respondido à pergunta.

Fonte: Relatório de Pesquisa de Satisfação com Cidadãos Usuários e não Usuários do SUS quanto aos aspectos de acesso e qualidade percebida na atenção a saúde- Departamento de Ouvidoria Geral do SUS (DOGES, 2012).

Os encaminhamentos para outros procedimentos no SUS, também foram elencados, sendo que $49 \%$ dos usuários das UBS informaram terem sido encaminhados. Destes, $60 \%$ conseguiram realizar exames, $56 \%$ consultaram com especialista, $76 \%$ tiveram que ser internados e $73 \%$ acessaram o serviço de urgência.

Quanto ao acesso aos medicamentos prescritos, $57 \%$ dos entrevistados os obteve na própria UBS, para 7\% foram disponibilizados gratuitamente em outros serviços no SUS, para outros $7 \%$ os medicamentos foram disponibilizados pela farmácia popular, $19 \%$ comprou na rede privada e $10 \%$ não conseguiu a medicação, o que merece três considerações: é expressivo o número de usuários que conseguem a medicação necessária na própria UBS sem precisar se deslocar; também é importante o número de usuários (71\%) que adquirem os medicamentos gratuitos ou subsidiados; mas não é desprezível o percentual (29\%) de usuários que ficaram sem o medicamento prescrito ou tiveram que comprá-lo.

\section{Eixo 3- Avaliação geral do SUS}

Para compor esse último eixo, agregaram-se os dados dos que utilizaram os serviços do SUS nos últimos 12 meses e os que referiam não ter buscado. Entre aqueles que buscaram algum serviço, $36 \%$ atribuíram o conceito de muito bom ou bom, $34 \%$ de regular, seguido de $29 \%$ que consideraram como ruim ou muito ruim. No segundo, composto por entrevistados que não acessaram nenhum serviço do SUS nos últimos 12 meses os percentuais ficaram em $25 \%$ com avaliação muito boa ou boa, $30 \%$ como regular e $45 \%$ como ruim ou muito ruim.

Os dados expressam uma diferenciação na avaliação entre os dois grupos, à medida que a proporção de opiniões de que os serviços do SUS são muito bons ou bons foi maior (36\%) entre os que utilizaram, do que entre o outro grupo (25\%). Outro achado interessante é de que a proporção de opiniões de que os serviços prestados pelo SUS são ruins ou muito ruins foi maior entre os entrevistados que não tiveram experiência com algum dos serviços pesquisados (45\%), em comparação com aqueles que tiveram (29\%). 


\section{Algumas reflexões}

Um primeiro efeito da pesquisa é colocar interrogações sobre quem são os usuários do SUS. Mais que uma provocação, a forma de agrupar quem utilizou algum serviço de saúde do SUS, no recorte de 12 meses, vem problematizar que o imaginário coletivo reconhece "inadequadamente" como usuários do sistema, apenas aqueles que não possuem plano de saúde privado. Considerando o escopo de ações e serviços ofertados pelo SUS, pode-se afirmar que todas as pessoas são usuárias do sistema, pois suas ações vão além das assistenciais, abarcam entre outras, oferta de medicamentos, ações de vigilância à saúde, normatização e fiscalização das condições sanitárias de produção, armazenamento e distribuição dos alimentos e dos estabelecimentos comerciais e produtivos.

Sobre isso, um estudo ${ }^{8}$, ao fazer um balanço dos 20 anos da Reforma Sanitária, reflete que houve, durante um período, um deslocamento dos temas das produções técnicas e acadêmicas, visto que no início eram mais voltadas para questões mais abrangentes como democracia e papel do estado. Neste deslocamento, houve muitas confusões entre termos e significados e por outro lado, a criação de termos novos, muitos deles para contrapor o ideário da Reforma Sanitária, a exemplo do termo SUS - dependente. Nesse sentido, concorda-se com a autora de que este termo "remete exatamente à exclusão social, uma vez que se refere àqueles sujeitos sociais que não tem acesso à atenção a saúde que não seja por meio dos serviços públicos de saúde." ${ }^{8}$

Os dados revelaram que existem desigualdades socioeconômicas importantes entre os dois grupos, sendo os que referiram utilizar os serviços do SUS, menos favorecidos. No entanto, é preciso considerar que existe uma parcela significativa de usuários com planos de saúde que buscaram o atendimento no SUS. Tal achado permite presumir que muitos usuários, inclusive de planos de saúde, estão encontrando no SUS serviços capazes de lhes ofertar cuidado de qualidade e que por isso os acionam.

Outro ponto que merece ser discutido no eixo "quem são os usuários do SUS?" é o maior percentual de mulheres que referiram utilizar os serviços de saúde. Ao investigar as razões para a não procura masculina, estudos evidenciaram justificativas que estão implicadas com a forma de organização dos serviços, por exemplo, horário de atendimento coincidir com o trabalho, e com as barreiras socioculturais que atribuem ao homem à falsa premissa que "são invulneráveis." "9,10,11

Pode-se acrescentar que o SUS vem investindo fortemente em políticas direcionadas a grupos específicos, considerando as particularidades e especificidades de cada um. Como exemplos, podem-se citar a Política Nacional de Atenção Integral a Saúde do Homem ${ }^{11}$ e a previsão das Unidades Básicas de Saúde Fluviais, Equipes de Saúde Ribeirinha e Consultórios na Rua na $\mathrm{PNAB}^{2}$ como Equipes de Atenção Básica que devem buscar e ofertar atenção integral a estas populações que reconhecem o potencial da $A B$ no cuidado e reforçam a ideia de um sistema de saúde público e de qualidade para todos os brasileiros.

Os dados apresentados no eixo "A Atenção Básica como referência de cuidado" confirmam que as Unidades Básicas de Saúde são, com larga vantagem, os serviços mais acessíveis e acessados do SUS. A pesquisa mostra que as pessoas usam o serviço, que ele está localizado próximo de suas casas e que os profissionais chegam até a população em seu próprio domicílio. Também permitem inferir que quem é coberto de fato está usando o serviço e que as pessoas sabem cada vez mais identificar quando uma UBS se organiza pela ESF. 
Com relação à satisfação com os profissionais da $A B$ faz-se necessário alguns apontamentos, como por exemplo, que é incorreto deduzir que a qualidade do atendimento dos ACS é melhor que a dos enfermeiros, embora esteja claro que as pessoas estão mais satisfeitas com os primeiros do que com os últimos. Neste contexto, é preciso considerar que talvez a satisfação esteja mais relacionada com o acesso àquilo que se buscou, e com a realização ou não de algumas das expectativas que o usuário levou para esse atendimento, do que com a própria qualidade de cuidado ofertado.

Parece razoável supor que pesou na avaliação da enfermagem a impossibilidade de ofertar certos procedimentos que motivaram a maioria das buscas pelos serviços. Por exemplo, $79 \%$ dos usuários disseram ter buscado no SUS uma consulta médica, 53\% medicamentos e $49 \%$ exames, ações que dependem, na maioria dos casos, diretamente do profissional médico. Outro grupo representado por $45 \%$ dos entrevistados buscou vacinações, curativos e orientações. Talvez um estudo que identificasse a existência de diferenças entre esses grupos quanto à avaliação de enfermagem traria mais subsídios para a discussão, pois é preciso considerar que a enfermagem teve mais governabilidade de ofertar ações que atenderam à demanda para o segundo grupo do que para o primeiro.

Obter acesso (entrar nos serviços de saúde) não garante que as necessidades serão satisfeitas, e mais, acessibilidade refere-se às facilidades e dificuldades em buscar/receber cuidado (interação entre quem busca e quem oferece cuidado). ${ }^{3}$ Assim, define acessibilidade como uma prática não normativa, de inter-relação e intersubjetividades, portanto uma prática que significa não apenas no acesso dos usuários aos serviços de saúde, mas também a resolução das suas demandas e necessidades de saúde. Todavia, assinalase que, muitas vezes, o usuário pode não ter acesso ao que "foi buscar", seja por ter mudado a compreensão sobre essa necessidade, seja por não haver disponibilidade no serviço naquela ocasião e, mesmo assim, sair satisfeito com os profissionais por ter se sentido acolhido e cuidado. $^{3}$

Ao comentar os achados referentes ao agendamento de consulta e tempo de espera na unidade é importante enfatizar que não se quer estabelecer parâmetros únicos para realidades diferentes $e$ complexas. Pode-se dizer que de um modo geral, é expressivo o número de usuários que conseguem atendimento em tempo oportuno, o que mostra as unidades de saúde mais preparadas para ser de fato uma porta de entrada que se molda em função da necessidade dos usuários. De outro lado, aguardar três ou quatro semanas para marcar uma consulta, independente da necessidade de saúde em questão, é um tempo muito grande. Também não é razoável esperar mais do que 2 horas numa UBS para um atendimento. Nesse sentido, os dados reforçam que a $A B$ ainda apresenta aspectos importantes a serem melhorados, além disso, apontam lacunas nas redes de atenção com relação ao fluxo de encaminhamentos. Cabe destacar que este tempo de espera pode ser reduzido com o uso de dispositivos de organização de agenda como marcação por hora e poderia ser aproveitado para ações de orientação, educação em saúde e intersetoriais, o que raramente acontece.

As ofertas de cuidados programados e prioritários na $A B$, como gestantes, crianças até dois anos, pessoas em condições crônicas, muitas vezes, são questionamentos realizados pelos usuários e acabam gerando desconforto nas equipes, pois aqueles que nesses grupos se encaixam conseguem acesso relativamente fácil. Em contrapartida, outros que não se "encaixam" 
em nenhum "grupo", mesmo estando em situações de sofrimento e apresentando um problema agudo, podem encontrar muita dificuldade para serem atendidos, exigindo incursões de madrugada em busca de uma das poucas fichas disponibilizadas "para o dia".

De outro lado, sabe-se que uma UBS pouco acessível e que não acolhe as pessoas nas situações agudas e de urgência, justamente na hora que se sentem mais aflitas e desamparadas, não é legitimada pela população e faz com que muitas pessoas busquem serviços de pronto atendimento com necessidades de saúde que seriam melhor acolhidas, cuidadas e acompanhadas nas UBS. Contudo, essa pressão pode exigir de uma UBS um volume de ações que extrapole a capacidade de oferta frente a uma demanda comprimida no tempo por diversos motivos que vão desde a insegurança do usuário (que se traduz num "não deixar pra amanhã, pois, amanhã não sei se terei oportunidade") à reprodução mais geral do consumismo e da instantaneidade da sociedade atual no conjunto das relações.

No que corresponde o eixo "Avaliação geral do SUS" reforça-se a ideia de que as pessoas que usam o SUS o avaliam a partir de suas vivências e experiências com os serviços do Sistema, ao passo que as pessoas que não usam tendem a reproduzir uma opinião muito influenciada pela representação social dominante, continuamente reiterada pelos meios de comunicação comerciais e de massa.

Tem-se notado que os elementos negativos sobre o SUS é que ganham visibilidade na grande imprensa comercial. De fato, matérias que relatam os sofrimentos dos cidadãos no uso do SUS são mais atrativas e tem mais audiência, elemento central para uma mídia comercial que além de vender anúncios muitas vezes funciona como caixa de reverberação de interesses comerciais, econômicos e políticos colocados no campo do debate e disputa do público e do privado, do mercado da saúde no Brasil e das relações entre saúde e sociedade.

\section{Algumas considerações}

Os estudos constroem-se
sobre outros estudos, não no
sentido de que retornam onde
outros deixaram, mas porque
melhor informados e melhor
conceituados, eles mergulham
mais profundamente nas mesmas
coisas. ${ }^{22}$

Com as considerações de Geertz ${ }^{12}$, pontua-se que os dados compartilhados são resultantes de uma primeira aproximação e que novos inquéritos mostram-se necessários para qualificação do cuidado e fortalecimento da participação social. Desse modo, entende-se que a pesquisa não se esgota aqui.

O panorama elucidado mostra avanços significativos em relação ao SUS, e em especial, ao que corresponde a $A B$. Os dados mostram que o perfil dos usuários que buscam o SUS está se modificando, à medida que mais da metade dos entrevistados com plano de saúde referiu buscar atendimento no SUS. Nesse sentido, pode-se apostar na desconstrução do ideário do SUS como alternativa para pobres, tendo em vista a maior divulgação do escopo de ações e de experiências positivas no Sistema. Talvez um caminho seja trabalhar mais o lema "todos usam o SUS".

Partindo para avaliação na perspectiva dos usuários, elencam-se como pontos positivos a utilização da $A B$, a satisfação com os profissionais que ali atuam e a distribuição das unidades no território. De outro lado, há pontos a serem considerados como passíveis de melhoria, inclusive 
levando-se em conta as condições que já estão dadas, como o tempo de espera para agendamento de consultas, de espera na unidade e o percentual de usuários que não conseguiu obter medicação gratuita na unidade ou na rede.

Outra questão abordada no estudo que merece um destaque é a diferença de avaliação do SUS entre quem utilizou e quem não o utilizou. Será que as pesquisas de avaliação ao não separarem estes dois grupos não estão produzindo fenômenos diferentes e apresentando-o como um todo? Não seria interessante questionar o que leva estes usuários que não usaram o SUS a avaliarem-no como ruim ou muito ruim? Questões como essas merecem ser exploradas, assim como, abordagens qualitativas são bem vindas, na medida em que poderiam contribuir para a melhoria dos processos avaliativos.

Por fim, cabe destacar a potência da Ouvidoria ativa no fortalecimento do SUS e na operacionalização dos seus princípios, diretrizes e serviços.

\section{Referências}

1. Silva Junior AG, Mascarenhas MTM. Avaliação da Atenção Básica em Saúde sob a ótica da Integralidade: aspectos conceituais e metodológicos. In: Pinheiro R, Mattos RA. Cuidado: as fronteiras da integralidade. Rio de Janeiro: CEPESC-IMS/UERJ/ABRASCO. 2008; p.243-260.

2. Brasil. Ministério da Saúde. Portaria GM no 1.654, de 21 de outubro de 2011. Aprova a Política Nacional de Atenção Básica, estabelecendo a revisão de diretrizes e normas para a organização da Atenção Básica, para a Estratégia Saúde da Família (ESF) e o Programa de Agentes Comunitários de Saúde (PACS). Brasília, 2011.

${ }^{3}$. Gerhardt TE et al. Determinantes sociais e práticas avaliativas de integralidade em saúde: pensando a situação de adoecimento crônico em um contexto rural. In: Pinheiro R, Martins PH (Orgs). Avaliação em saúde na perspectiva do usuário: abordagem multicêntrica. Rio de Janeiro: CEPESC / IMS-UERJ; Recife: Editora Universitária UFPE; São Paulo: ABRASCO; 2009; p.287-98.

4. Esperidião MA, Trad LAB. Avaliação de satisfação de usuários: considerações teóricoconceituais. Cadernos de Saúde Pública, Rio de Janeiro, 2006; 22(6):1267-1276.

5. Bolzan LC et al. Ouvidoria ativa: a inovação das pesquisas de satisfação na ouvidoria-geral do SUS. In: Congresso Consad de Gestão Pública (5: Brasília: 2012) [Anais.] Brasília: 2012.

${ }^{6}$. Brasil. Ministério da Saúde. Relatório Preliminar da Pesquisa de Satisfação com Cidadãos Usuários e não Usuários do SUS. Brasília, 2012.

7. Brasil. Ministério da Saúde. Portaria no 648, de 28 de março de 2006. Aprova a Política Nacional de Atenção Básica, estabelecendo a revisão de diretrizes e normas para a organização da Atenção Básica para o Programa Saúde da Família (PSF) e o Programa Agentes Comunitários de Saúde (PACS). Brasília, 2006.

${ }^{8}$. Cohn A. A Reforma Sanitária Brasileira Após 20 anos do SUS: reflexões. Cadernos de Saúde Pública, Rio de Janeiro, 2009; 25(7): 1614-1619. 


\section{Artigo Original}

9. Araújo MAL, Leitão GCM. Acesso à consulta a portadores de doenças sexualmente transmissíveis: experiências de homens em uma unidade de saúde de Fortaleza, Ceará, Brasil. Cadernos de Saúde Pública, Rio de Janeiro, 2005; 21(2): 396-404.

${ }^{10}$. Gomes R, Nascimento EF, Araújo FC. Por que os homens buscam menos os serviços de saúde do que as mulheres? As explicações de homens com baixa escolaridade e homens com ensino superior. Cadernos de Saúde Pública, 2007: 23(3): 565-74.

11. Brasil. Ministério da Saúde. Secretaria de Atenção à Saúde. Política Nacional de Atenção Integral à saúde do homem. Brasília, 2008.

12. Geertz C. A interpretação das culturas. Rio de Janeiro: LTC, 1989. 